\title{
Supplemental Info
}

\section{Discovery of the first selective $\mathrm{M}_{4}$ muscarinic acetylcholine receptor antagonists with in vivo anti- parkinsonian and anti-dystonic efficacy}

Mark S. Moehle ${ }^{1,2}$, Aaron M. Bender ${ }^{1}$, Jonathan W. Dickerson ${ }^{1}$, Daniel J. Foster ${ }^{1,3}$, Aidong Qi ${ }^{l}$, Hyekyung P. Cho, Yuping Donsante ${ }^{4}$, Weimin Peng ${ }^{1}$, Zoey Bryant ${ }^{1}$, Kaylee J. Stillwell ${ }^{1}$, Thomas M. Bridges ${ }^{1}$, Sichen Chang ${ }^{1}$, Katherine J. Watson ${ }^{1}$, Jordan C. O'Neill', Julie L. Engers ${ }^{1}$, Li Peng', Alice L. Rodriguez ${ }^{1}$, Colleen M. Niswender ${ }^{1,3}$, Craig W. Lindsley ${ }^{l}$, Ellen J. Hess ${ }^{4}$, P. Jeffrey Conn ${ }^{1,3 *}$, and Jerri M. Rook ${ }^{1 *}$.

${ }^{1}$ Department of Pharmacology, Warren Center for Neuroscience Drug Discovery, Vanderbilt University, Nashville, TN, 37232, United States

${ }^{2}$ Department of Pharmacology \& Therapeutics, Center for Translational Research in Neurodegeneration, University of Florida, Gainesville, FL, 32610, United States

${ }^{3}$ Vanderbilt Kennedy Center, Vanderbilt University, Nashville, TN, 37232, United States

4 Department of Pharmacology \& Chemical Biology, Emory University, Atlanta, Georgia, 30322, United States

\section{*Corresponding Authors}

Jerri M. Rook: Department of Pharmacology, Warren Center for Neuroscience Drug Discovery, Vanderbilt University, Nashville, TN, 37232, United States. Email: jerri.m.rook@vanderbilt.edu

P. Jeffrey Conn: Department of Pharmacology, Warren Center for Neuroscience Drug Discovery, and Vanderbilt Kennedy Center Vanderbilt University, Nashville, TN, 37232, United States. Email: Jeffrey.conn@vanderbilt.edu 


\section{Table of Contents}

Scheme S1. Chemistry Supporting Information............................... S-3 to S-6

Table S1. Radioligand binding data for stable cell lines..............................

Table S2. Ancillary Pharmacology of VU6021625 ............................ 8 to S-11

Figure S1. VU6021625 does not alter spontaneous locomotion..............................S-12

Figure S2. Wildtype and $\mathrm{M}_{4}$ knockout mouse haloperidol induced catalepsy...............S-13 
<smiles>CC(C)(C)OC(=O)N1C[C@H]2C[C@H](Nc3ccc(Cl)nn3)C[C@H]2C1</smiles>

tert-butyl (3aR,5s,6aS)-5-((6-chloropyridazin-3-yl)amino)hexahydrocyclopenta[c]pyrrole2(1H)-carboxylate (3). Tert-butyl $(3 \mathrm{a} R, 5 \mathrm{~s}, 6 \mathrm{a} S)$-5-aminohexahydrocyclopenta[c]pyrrole-2(1H)carboxylate $(5.0 \mathrm{~g}, 22.1 \mathrm{mmol}, 1 \mathrm{eq})$ and 3,6-dichloropyridazine $(9.87 \mathrm{~g}, 66.3 \mathrm{mmol}, 3 \mathrm{eq})$ were combined in tert-butanol $(30 \mathrm{~mL})$, and DIPEA $(11.5 \mathrm{~mL}, 66.3 \mathrm{mmol}, 3 \mathrm{eq})$ was added. The resulting solution was heated to $150{ }^{\circ} \mathrm{C}$ under microwave irradiation for $2 \mathrm{~h}$, after which time the reaction mixture was concentrated under reduced pressure, and crude residue was purified by column chromatography (3-100\% EtOAc in hexanes) to give the title compound as a white solid (4.87 g, 65\%).

${ }^{1} \mathrm{H}-\mathrm{NMR}(400 \mathrm{MHz}, \mathrm{MeOD}) \delta 7.27(\mathrm{~d}, J=9.4 \mathrm{~Hz}, 1 \mathrm{H}), 6.87(\mathrm{~d}, J=9.4 \mathrm{~Hz}, 1 \mathrm{H}), 4.41(\mathrm{p}, J=6.3$ $\mathrm{Hz}, 1 \mathrm{H}), 3.55(\mathrm{dd}, J=11.1,8.0 \mathrm{~Hz}, 2 \mathrm{H}), 3.19(\mathrm{dd}, J=11.4,3.8 \mathrm{~Hz}, 2 \mathrm{H}), 2.90-2.80(\mathrm{~m}, 2 \mathrm{H}), 1.90$ $-1.92(\mathrm{~m}, 2 \mathrm{H}), 1.89-1.81(\mathrm{~m}, 2 \mathrm{H}), 1.46(\mathrm{~s}, 9 \mathrm{H})$.

${ }^{13} \mathrm{C}-\mathrm{NMR}(101 \mathrm{MHz}, \mathrm{MeOD}) \delta 159.3,156.3,146.8,130.3,120.5,80.8,53.7,53.2$ (signal broadening is observed) 42.3 (signal broadening is observed), 39.5, 28.8.

ES-MS $[\mathrm{M}+\mathrm{H}]^{+}=283.2$ (- t-butyl).

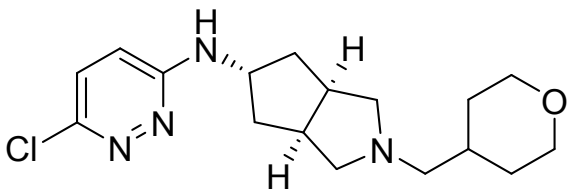

\section{$(3 \mathrm{a} R, 5 \mathrm{~s}, 6 \mathrm{aS})-\mathrm{N}$-(6-chloropyridazin-3-yl)-2-((tetrahydro-2H-pyran-4-}

yl)methyl)octahydrocyclopenta[c]pyrrol-5-amine $\quad$ (5). Tert-butyl $\quad(3 \mathrm{a} R, 5 \mathrm{~s}, 6 \mathrm{a} S)-5-((6-$ chloropyridazin-3-yl)amino)hexahydrocyclopenta[c]pyrrole-2(1H)-carboxylate (3) (4.86 g, 14.3 mmol, 1 eq) was dissolved in 1,4-dioxane $(70 \mathrm{~mL})$ and $\mathrm{MeOH}(20 \mathrm{~mL})$, and $4 \mathrm{M} \mathrm{HCl}$ in dioxanes solution $(50 \mathrm{~mL})$ was added dropwise. The resulting solution was stirred at r.t. for $1 \mathrm{~h}$, after which time solvents were concentrated under reduced pressure to give the $\mathrm{HCl}$ salt as a white solid, which was dried under vacuum and used without additional purification (3.95 g, 100\%). The $\mathrm{HCl}$ salt was then suspended in DCM $(40 \mathrm{~mL})$ and THF $(50 \mathrm{~mL})$, and tetrahydro-2H-pyran-4-carbaldehyde (2.29 g, $20.1 \mathrm{mmol}, 1.4 \mathrm{eq})$ was added, followed by sodium triacetoxyborohydride $(6.08 \mathrm{~g}, 28.9$ mmol, $2 \mathrm{eq}$ ). The resulting solution was stirred at r.t. for $1.5 \mathrm{~h}$, after which time the reaction mixture was quenched with sat. $\mathrm{NaHCO}_{3}$, and extracted with DCM. Combined organic extracts were washed with brine, and dried over $\mathrm{MgSO}_{4}$. Solvents were filtered and concentrated to give the title compound as a white solid (4.31 g, 89\% over 2 steps).

${ }^{1} \mathrm{H}-\mathrm{NMR}(400 \mathrm{MHz}, \mathrm{MeOD}) \delta 7.26(\mathrm{~d}, J=9.4 \mathrm{~Hz}, 1 \mathrm{H}), 6.86(\mathrm{~d}, J=9.4 \mathrm{~Hz}, 1 \mathrm{H}), 4.43-4.36$ (m, $1 \mathrm{H}), 3.93(\mathrm{dd}, J=11.3,3.7 \mathrm{~Hz}, 2 \mathrm{H}), 3.42(\mathrm{td}, J=11.9,1.9 \mathrm{~Hz}, 2 \mathrm{H}), 2.84-2.68(\mathrm{~m}, 4 \mathrm{H}), 2.31$ (d, $J=6.8 \mathrm{~Hz}, 2 \mathrm{H}), 2.27-2.17(\mathrm{~m}, 2 \mathrm{H}), 1.91(\mathrm{ddd}, J=12.9,5.9,2.1 \mathrm{~Hz}, 2 \mathrm{H}), 1.83-1.64(\mathrm{~m}, 5 \mathrm{H})$, $1.32-1.21(\mathrm{~m}, 2 \mathrm{H})$. 
${ }^{13} \mathrm{C}-\mathrm{NMR}(101 \mathrm{MHz}, \mathrm{MeOD}) \delta 159.7,146.6,130.3,120.4,68.9,63.5,62.9,53.4,41.5,39.1,35.3$, 32.9 .

ES-MS $[\mathrm{M}+\mathrm{H}]^{+}=337.2$.<smiles>Fc1ccc(Cl)c(-c2ccc(NC3C[C@@H]4CN(CC5CCOCC5)C[C@H]4C3)nn2)c1</smiles>

(3aR,5s,6aS)- $N$-(6-(2-chloro-5-fluorophenyl)pyridazin-3-yl)-2-((tetrahydro-2H-pyran-4yl)methyl)octahydrocyclopenta[c]pyrrol-5-amine $\quad(6, \quad$ VU6013720 $) \quad(3 \mathrm{a} R, 5 \mathrm{~s}, 6 \mathrm{a} S)-N-(6-$ chloropyridazin-3-yl)-2-((tetrahydro-2H-pyran-4-yl)methyl)octahydrocyclopenta[c]pyrrol-5amine (5) (100 mg, $0.30 \mathrm{mmol}, 1 \mathrm{eq}), 2$-chloro-5-fluorophenylboronic acid (62 mg, $0.36 \mathrm{mmol}$, $1.2 \mathrm{eq}$ ), potassium carbonate $(125 \mathrm{mg}, 0.89 \mathrm{mmol}, 3 \mathrm{eq}$ ) and BrettPhos-Pd-G3 (27 mg, 0.030 mmol, 0.1 eq) were combined in a vial, and 5:1 1,4-dioxane $/ \mathrm{H}_{2} \mathrm{O}$ solution $(5 \mathrm{~mL}$ total, degassed under vacuum) was added via syringe. The resulting mixture was stirred under an inert atmosphere at $100{ }^{\circ} \mathrm{C}$ for $2.5 \mathrm{~h}$, after which time the reaction mixture was cooled to r.t. and diluted with water and DCM. The aqueous layer was extracted with DCM, and combined organic extracts were filtered through a phase separator and concentrated. Crude residue was purified by RP-HPLC (10$50 \% \mathrm{MeCN}$ in $0.1 \%$ TFA aqueous solution over $20 \mathrm{~min}$ ). Fractions containing product were basified with sat. $\mathrm{NaHCO}_{3}$, and extracted with DCM. Combined organic extracts were dried over $\mathrm{MgSO}_{4}$, filtered and concentrated under reduced pressure to give the title compound as a white solid (40 mg, 31\%).

${ }^{1} \mathrm{H}-\mathrm{NMR}(400 \mathrm{MHz}, \mathrm{MeOD}) \delta 7.57-7.52(\mathrm{~m}, 2 \mathrm{H}), 7.35$ (dd, $\left.J=9.0,3.1 \mathrm{~Hz}, 1 \mathrm{H}\right), 7.20$ (ddd, $J=$ 8.8, 7.9, $3.1 \mathrm{~Hz}, 1 \mathrm{H}), 6.91(\mathrm{~d}, J=9.3 \mathrm{~Hz}, 1 \mathrm{H}), 4.56-4.48(\mathrm{~m}, 1 \mathrm{H}), 3.94(\mathrm{dd}, J=11.2,3.5 \mathrm{~Hz}$, 2H), $3.43(\mathrm{td}, J=11.9,1.9 \mathrm{~Hz}, 2 \mathrm{H}), 2.87-2.74(\mathrm{~m}, 4 \mathrm{H}), 2.34(\mathrm{~d}, J=6.9 \mathrm{~Hz}, 2 \mathrm{H}), 2.26(\mathrm{dd}, J=$ 8.4, $4.0 \mathrm{~Hz}, 2 \mathrm{H}), 1.97$ (ddd, $J=12.9,5.9,2.1 \mathrm{~Hz}, 2 \mathrm{H}), 1.84-1.70(\mathrm{~m}, 5 \mathrm{H}), 1.34-1.22(\mathrm{~m}, 2 \mathrm{H})$.

${ }^{13} \mathrm{C}-\mathrm{NMR}(101 \mathrm{MHz}, \mathrm{MeOD}) \delta 162.8(\mathrm{~d}, J=246.1 \mathrm{~Hz}), 159.7,150.95(\mathrm{~d}, J=1.9 \mathrm{~Hz}), 139.8(\mathrm{~d}, J$ $=8.1 \mathrm{~Hz}), 132.8(\mathrm{~d}, J=8.5 \mathrm{~Hz}), 130.5,128.6(\mathrm{~d}, J=3.3 \mathrm{~Hz}), 118.9(\mathrm{~d}, J=24.1 \mathrm{~Hz}), 118.0(\mathrm{~d}, J=$ $23.0 \mathrm{~Hz}), 116.2,68.9,63.6,63.0,53.3,41.5,39.3,35.3,33.0$.

ES-MS $[\mathrm{M}+\mathrm{H}]^{+}=431.4$.<smiles>Cn1cc2cc(-c3ccc(N[C@@H]4C[C@@H]5CN(CC6CCOCC6)C[C@@H]5C4)nn3)ccc2n1</smiles> 
(3aR,5s,6aS)- $N$-(6-(2-methyl-2H-indazol-5-yl)pyridazin-3-yl)-2-((tetrahydro-2H-pyran-4yl)methyl)octahydrocyclopenta[c]pyrrol-5-amine $\quad(7, \quad$ VU6021625). $\quad(3 \mathrm{a} R, 5 \mathrm{~s}, 6 \mathrm{a} S)-\mathrm{N}-(6-$ chloropyridazin-3-yl)-2-((tetrahydro-2H-pyran-4-yl)methyl)octahydrocyclopenta[c]pyrrol-5amine (5) (1.0 g, $2.97 \mathrm{mmol}, 1 \mathrm{eq})$, 2-methylindazole-5-boronic acid pinacol ester (996 mg, 3.86 mmol, $1.3 \mathrm{eq})$, potassium carbonate $(1.25 \mathrm{~g}, 8.91 \mathrm{mmol}, 3 \mathrm{eq})$ and BrettPhos-Pd-G3 (269 mg, 0.30 mmol, 0.1 eq) were combined in a vial, and 5:1 1,4-dioxane $/ \mathrm{H}_{2} \mathrm{O}$ solution $(15 \mathrm{~mL}$ total, degassed under vacuum) was added via syringe. The resulting mixture was stirred under an inert atmosphere at $100{ }^{\circ} \mathrm{C}$ for $3 \mathrm{~h}$, after which time the reaction mixture was cooled to r.t. and diluted with water and DCM. The aqueous layer was extracted with DCM, and combined organic extracts were dried over $\mathrm{MgSO}_{4}$, filtered and concentrated under reduced pressure. Crude residue was purified by RPHPLC $\left(20-60 \% \mathrm{MeCN}\right.$ in $0.05 \% \mathrm{NH}_{4} \mathrm{OH}$ aqueous solution over $\left.20 \mathrm{~min}\right)$. Fractions containing product were concentrated to give the title compound as a white solid (431 mg, 34\%).

${ }^{1} \mathrm{H}-\mathrm{NMR}(400 \mathrm{MHz}, \mathrm{MeOD}) \delta 8.27$ (s, 1H), 8.17 (dd, $\left.J=1.7,0.9 \mathrm{~Hz}, 1 \mathrm{H}\right), 7.95(\mathrm{dd}, J=9.1,1.7$ $\mathrm{Hz}, 1 \mathrm{H}), 7.78$ (d, $J=9.4 \mathrm{~Hz}, 1 \mathrm{H}), 7.67$ (dt, $J=9.1,1.0 \mathrm{~Hz}, 1 \mathrm{H}), 6.93$ (d, $J=9.4 \mathrm{~Hz}, 1 \mathrm{H}), 4.54-$ $4.47(\mathrm{~m}, 1 \mathrm{H}), 4.23(\mathrm{~s}, 3 \mathrm{H}), 3.94$ (dd, $J=11.0,3.4 \mathrm{~Hz}, 2 \mathrm{H}), 3.43(\mathrm{td}, J=11.9,2.0 \mathrm{~Hz}, 2 \mathrm{H}), 2.91-$ $2.73(\mathrm{~m}, 4 \mathrm{H}), 2.36(\mathrm{~d}, J=6.9 \mathrm{~Hz}, 2 \mathrm{H}), 2.30-2.26(\mathrm{~m}, 2 \mathrm{H}), 2.00-1.95(\mathrm{~m}, 2 \mathrm{H}), 1.85-1.70(\mathrm{~m}$, $5 \mathrm{H}), 1.33-1.22(\mathrm{~m}, 2 \mathrm{H})$.

${ }^{13} \mathrm{C}-\mathrm{NMR}(101 \mathrm{MHz}, \mathrm{MeOD}) \delta 159.3,152.5,150.1,131.9,127.4,127.3,126.3,123.6,119.0$, $117.8,68.9,63.5,62.9,53.3,41.5,40.3,39.2,35.2,32.9$. Note: 1 aromatic signal is obscured.

ES-MS $[\mathrm{M}+\mathrm{H}]^{+}=433.0$.

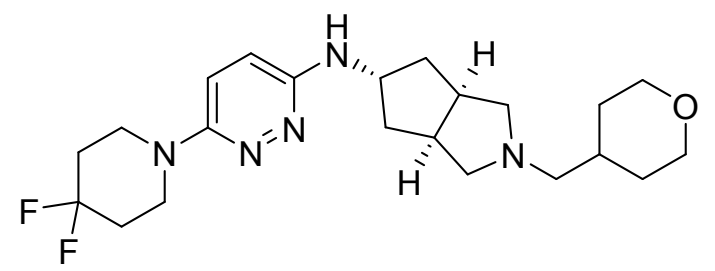

(3aR,5s,6aS)- $N$-(6-(4,4-difluoropiperidin-1-yl)pyridazin-3-yl)-2-((tetrahydro-2H-pyran-4yl)methyl)octahydrocyclopenta[c]pyrrol-5-amine $\quad(\mathbf{8}, \quad$ VU6021302). $\quad(3 \mathrm{a} R, 5 \mathrm{~s}, 6 \mathrm{a} S)-N-(6-$ chloropyridazin-3-yl)-2-((tetrahydro-2H-pyran-4-yl)methyl)octahydrocyclopenta[c]pyrrol-5amine (5) (1.0 g, $2.96 \mathrm{mmol}, 1 \mathrm{eq})$ and 4,4-difluoropiperidine hydrochloride (4.68 g, $29.7 \mathrm{mmol}$, $10 \mathrm{eq})$ were combined in NMP $(10 \mathrm{~mL})$, and DIPEA $(5.17 \mathrm{~mL}, 29.7 \mathrm{mmol}, 10 \mathrm{eq})$ was added. The resulting solution was stirred under microwave irradiation at $200^{\circ} \mathrm{C}$ for $2 \mathrm{~h}$, after which time the reaction mixture was purified directly by RP-HPLC $\left(25-65 \% \mathrm{MeCN}\right.$ in $0.05 \% \mathrm{NH}_{4} \mathrm{OH}$ aqueous solution over $20 \mathrm{~min}$ ). Fractions containing product were concentrated to give the title compound as a slightly tan solid (797 $\mathrm{mg}, 64 \%)$.

${ }^{1} \mathrm{H}-\mathrm{NMR}(400 \mathrm{MHz}, \mathrm{MeOD}) \delta 7.18(\mathrm{~d}, J=9.7 \mathrm{~Hz}, 1 \mathrm{H}), 6.81(\mathrm{~d}, J=9.7 \mathrm{~Hz}, 1 \mathrm{H}), 4.36-4.29(\mathrm{~m}$, $1 \mathrm{H}), 3.93(\mathrm{dd}, J=11.1,3.4 \mathrm{~Hz}, 2 \mathrm{H}), 3.57-3.54(\mathrm{~m}, 4 \mathrm{H}), 3.42(\mathrm{td}, J=11.8,2.0 \mathrm{~Hz}, 2 \mathrm{H}), 2.88-$ $2.86(\mathrm{~m}, 2 \mathrm{H}), 2.79-2.69(\mathrm{~m}, 2 \mathrm{H}), 2.34(\mathrm{~d}, J=6.9 \mathrm{~Hz}, 2 \mathrm{H}), 2.23(\mathrm{dd}, J=9.3,5.1 \mathrm{~Hz}, 2 \mathrm{H}), 2.08-$ $1.98(\mathrm{~m}, 4 \mathrm{H}), 1.91$ (ddd, $J=12.9,6.1,2.3 \mathrm{~Hz}, 2 \mathrm{H}), 1.83-1.62(\mathrm{~m}, 5 \mathrm{H}), 1.31-1.21(\mathrm{~m}, 2 \mathrm{H})$. 
${ }^{13} \mathrm{C}-\mathrm{NMR}(101 \mathrm{MHz}, \mathrm{MeOD}) \delta 155.9,155.7,123.3(\mathrm{t}, J=240.8 \mathrm{~Hz}), 120.8,120.7,68.9,63.5$, 62.9, 53.4, $45.2(\mathrm{t}, J=5.2 \mathrm{~Hz}), 41.4,39.2,35.2,34.3(\mathrm{t}, J=23.0 \mathrm{~Hz}), 32.9$.

ES-MS $[\mathrm{M}+\mathrm{H}]^{+}=422.5$.

Scheme S1. Chemistry Supporting Information. Additional synthesis, ${ }^{1} \mathrm{H}-\mathrm{NMR}$, and ${ }^{13} \mathrm{C}-\mathrm{NMR}$ details for intermediates and final compounds 


\begin{tabular}{|l|c|c|}
\hline & {$\left[{ }^{3} \mathrm{H}\right] \mathrm{NMS} \mathrm{Kd}(\mathrm{nM})$} & $\mathrm{Bmax}(\mathrm{fmol} / \mathrm{mg})$ \\
\hline ratM1-CHO & $0.088 \pm 0.013$ & $1305 \pm 208$ \\
\hline ratM2/Gqi5-CHO & $0.155 \pm 0.016$ & $2146 \pm 223$ \\
\hline ratM3-CHO & $0.077 \pm 0.007$ & $1126 \pm 123$ \\
\hline ratM4/Gqi5-CHO & $0.067 \pm 0.022$ & $2178 \pm 731$ \\
\hline ratM5-CHO & $0.235 \pm 0.041$ & $1701 \pm 258$ \\
\hline hM1-CHO & $0.075 \pm 0.004$ & $1479 \pm 129$ \\
\hline hM2/Gqi5-CHO & $0.114 \pm 0.012$ & $2089 \pm 561$ \\
\hline hM3-CHO & $0.116 \pm 0.014$ & $2233 \pm 737$ \\
\hline hM4/Gqi5-CHO & $0.041 \pm 0.004$ & $703 \pm 103$ \\
\hline hM5-CHO & $0.376 \pm 0.088$ & $2633 \pm 97$ \\
\hline
\end{tabular}

Table S1. Radioligand binding data for stable cell lines. Kd and Bmax values for cell lines stably expressing rat or human muscarinic receptor subtypes. Values are from at least 3 replicates. 


\begin{tabular}{|c|c|c|c|}
\hline Target & Radioligand & Species & \% Inhibition \\
\hline Adenosine A1 & [3H] DPCPX & Human & 12 \\
\hline Adenosine A2A & [3H] CGS-21680 & Human & 5 \\
\hline Adenosine A3 & [125I] AB-MECA & Human & 10 \\
\hline Adrenergic a1A & [3H] Prazosin & Rat & 13 \\
\hline Adrenergic a1B & [3H] Prazosin & Rat & 3 \\
\hline Adrenergic a1D & [3H] Prazosin & Human & 17 \\
\hline Adrenergic a $2 \mathrm{~A}$ & [3H] Rauwolscine & Human & 18 \\
\hline Adrenergic $ß 1$ & $\begin{array}{c}{[125 \mathrm{I}]} \\
\text { Cyanopindolol }\end{array}$ & Human & 6 \\
\hline Adrenergic $\beta 2$ & [3H] CGP-12177 & Human & 0 \\
\hline $\begin{array}{c}\text { Androgen } \\
\text { (Testosterone) }\end{array}$ & $\begin{array}{c}{[3 \mathrm{H}]} \\
\text { Methyltrienolone }\end{array}$ & Human & -13 \\
\hline Bradykinin B1 & $\begin{array}{l}\text { [3H] (Des-Arg10, } \\
\text { Leu9)- Kallidin }\end{array}$ & Human & -10 \\
\hline Bradykinin B2 & {$[3 \mathrm{H}]$ Bradykinin } & Human & -7 \\
\hline $\begin{array}{c}\text { Calcium Channel L- } \\
\text { Type, } \\
\text { Benzothiazepine }\end{array}$ & [3H] Diltiazem & Human & 7 \\
\hline $\begin{array}{c}\text { Calcium Channel L- } \\
\text { Type, } \\
\text { Dihydropyridine }\end{array}$ & {$[3 \mathrm{H}]$ Nitrendipine } & Rat & 1 \\
\hline $\begin{array}{c}\text { Calcium Channel N- } \\
\text { Type }\end{array}$ & $\begin{array}{c}\text { [125I] } \omega \text {-Conotoxin } \\
\text { GVIA }\end{array}$ & Rat & -9 \\
\hline Cannabinoid CB1 & {$[3 \mathrm{H}] \mathrm{SR} 141716 \mathrm{~A}$} & Rat & -3 \\
\hline Dopamine D1 & [3H] SCH-23390 & Human & 2 \\
\hline Dopamine D2S & [3H] Spiperone & Human & 17 \\
\hline Dopamine D3 & [3H] Spiperone & Human & 34 \\
\hline Dopamine D4.4 & [3H] Spiperone & Human & 3 \\
\hline
\end{tabular}




\begin{tabular}{|c|c|c|c|}
\hline Endothelin ETA & [125I] Endothelin-1 & Human & -11 \\
\hline Endothelin ETB & [125I] Endothelin-1 & Human & 2 \\
\hline $\begin{array}{l}\text { Epidermal Growth } \\
\text { Factor (EGF) }\end{array}$ & {$[125 \mathrm{I}] \mathrm{EGF}$} & Human & -5 \\
\hline Estrogen ERa & [3H] Estradiol & Human & -3 \\
\hline $\begin{array}{c}\text { GABAA, } \\
\text { Flunitrazepam, } \\
\text { Central }\end{array}$ & [3H] Flunitrazepam & Rat & -4 \\
\hline $\begin{array}{c}\text { GABAA, Muscimol, } \\
\text { Central }\end{array}$ & [3H] Muscimol & Rat & -11 \\
\hline GABAB1A & [3H] CGP-54626 & Human & 2 \\
\hline Glucocorticoid & $\begin{array}{c}{[3 \mathrm{H}]} \\
\text { Dexamethasone }\end{array}$ & Human & -2 \\
\hline Glutamate, Kainate & [3H] Kainic acid & Rat & 6 \\
\hline $\begin{array}{c}\text { Glutamate, NMDA, } \\
\text { Agonism }\end{array}$ & {$[3 \mathrm{H}] \mathrm{CGP}-39653$} & Rat & -5 \\
\hline $\begin{array}{c}\text { Glutamate, NMDA, } \\
\text { Glycine }\end{array}$ & [3H] MDL 105,519 & Rat & -12 \\
\hline $\begin{array}{c}\text { Glutamate, NMDA, } \\
\text { Phencyclidine }\end{array}$ & {$[3 \mathrm{H}] \mathrm{TCP}$} & Rat & -1 \\
\hline Histamine H1 & [3H] Pyrilamine & Human & 25 \\
\hline Histamine $\mathrm{H} 2$ & $\begin{array}{c}{[125 \mathrm{I}]} \\
\text { Aminopotentidine }\end{array}$ & Human & -3 \\
\hline Histamine H3 & $\begin{array}{c}{[3 \mathrm{H}] \mathrm{N}-\alpha-} \\
\text { Methylhistamine }\end{array}$ & Human & 88 \\
\hline $\begin{array}{l}\text { Imidazoline I2, } \\
\text { Central }\end{array}$ & [3H] Idazoxan & Rat & 2 \\
\hline Interleukin IL-1 R1 & $\begin{array}{c}{[125 \mathrm{I}] \text { Interleukin- }} \\
1 \beta\end{array}$ & Human & -7 \\
\hline $\begin{array}{c}\text { Leukotriene, } \\
\text { Cysteinyl CysLT1 }\end{array}$ & [3H] LTD4 & Human & 12 \\
\hline
\end{tabular}




\begin{tabular}{|c|c|c|c|}
\hline Melatonin MT1 & $\begin{array}{l}{[125 \mathrm{I}] 2-} \\
\text { Iodomelatonin }\end{array}$ & Human & -2 \\
\hline Muscarinic M1 & $\begin{array}{c}{[3 \mathrm{H}] \mathrm{N}-} \\
\text { Methylscopolamine }\end{array}$ & Human & 33 \\
\hline Muscarinic M2 & $\begin{array}{c}{[3 \mathrm{H}] \mathrm{N}-} \\
\text { Methylscopolamine }\end{array}$ & Human & 85 \\
\hline Muscarinic M3 & $\begin{array}{c}{[3 \mathrm{H}] \mathrm{N}-} \\
\text { Methylscopolamine }\end{array}$ & Human & 51 \\
\hline Neuropeptide Y Y1 & [125I] Peptide YY & Human & -13 \\
\hline Neuropeptide Y Y2 & [125I] Peptide YY & Human & -2 \\
\hline $\begin{array}{c}\text { Nicotinic } \\
\text { Acetylcholine } \alpha 1, \\
\text { Bungarotoxin }\end{array}$ & $\begin{array}{c}{[125 \mathrm{I}] \alpha-} \\
\text { Bungarotoxin }\end{array}$ & Human & -1 \\
\hline $\begin{array}{l}\text { Nicotinic } \\
\text { Acetylcholine } \alpha 3 \beta 4\end{array}$ & [125I] Epibatidine & Human & 55 \\
\hline $\begin{array}{c}\text { Opiate } \delta 1(\mathrm{OP} 1, \\
\text { DOP) }\end{array}$ & [3H] Naltrindole & Human & -1 \\
\hline Opiate $\kappa(\mathrm{OP} 2, \mathrm{KOP})$ & [3H] Diprenorphine & Human & 5 \\
\hline Opiate $\mu$ (OP3, MOP) & [3H] Diprenorphine & Human & 6 \\
\hline Phorbol Ester & {$[3 \mathrm{H}] \mathrm{PDBu}$} & Mouse & 9 \\
\hline $\begin{array}{l}\text { Platelet Activating } \\
\text { Factor (PAF) }\end{array}$ & {$[3 \mathrm{H}] \mathrm{PAF}$} & Human & -4 \\
\hline $\begin{array}{c}\text { Potassium Channel } \\
{[\text { KATP }]}\end{array}$ & [3H] Glyburide & Human & 11 \\
\hline $\begin{array}{c}\text { Potassium Channel } \\
\text { hERG }\end{array}$ & {$[3 \mathrm{H}]$ Astemizole } & Human & 23 \\
\hline Prostanoid EP4 & $\begin{array}{c}{[3 \mathrm{H}] \text { Prostaglandin }} \\
\text { E2 }\end{array}$ & Human & 0 \\
\hline Purinergic $\mathrm{P} 2 \mathrm{X}$ & $\begin{array}{c}{[3 \mathrm{H}] \alpha, \beta-} \\
\text { Methylene-ATP }\end{array}$ & Rat & 4 \\
\hline Rolipram & [3H] Rolipram & Rat & 0 \\
\hline
\end{tabular}


Serotonin (5-

Hydroxytryptamine)

5-HT1A

[3H] 8-OH-DPAT Human

6

Serotonin (5-

Hydroxytryptamine) [3H] Lysergic acid

5-HT2B

diethylamide

Human

53

Serotonin (5-

Hydroxytryptamine)

5-HT3

[3H] GR-65630

Human

$-2$

Sigma $\sigma 1$

[3H] Haloperidol

Human

38

Sodium Channel, Site

2

[3H]

\begin{tabular}{|c|c|c|c|}
\hline 2 & Batrachotoxinin & Rat & 15 \\
\hline Tachykinin NK1 & {$[3 \mathrm{H}]$ Substance P } & Human & -10 \\
\hline $\begin{array}{c}\text { Thyroid Hormone } \\
\text { Triiodothyronine }\end{array}$ & Rat & -21 \\
\hline $\begin{array}{c}\text { Transporter, } \\
\text { Dopamine (DAT) }\end{array}$ & {$[125 \mathrm{I}]$ RTI-55 } & Human & 15 \\
\hline $\begin{array}{c}\text { Transporter, GABA } \\
\text { Transporter, }\end{array}$ & {$[3 \mathrm{H}]$ GABA } & Rat & -5 \\
\hline $\begin{array}{c}\text { Norepinephrine } \\
\text { (NET) }\end{array}$ & {$[125 \mathrm{I}]$ RTI-55 } & Human & 15 \\
\hline $\begin{array}{c}\text { Transporter, } \\
\text { Serotonin }(5-\end{array}$ & & & \\
\hline $\begin{array}{c}\text { Hydroxytryptamine) } \\
\text { (SERT) }\end{array}$ & {$[3 \mathrm{H}]$ Paroxetine } & Human & -1 \\
\hline
\end{tabular}

Table S2. Ancillary Pharmacology of VU6021625. Eurofins panel showing binding of VU6021625 to a screen of 88 different receptors, transporters and enzymes. 

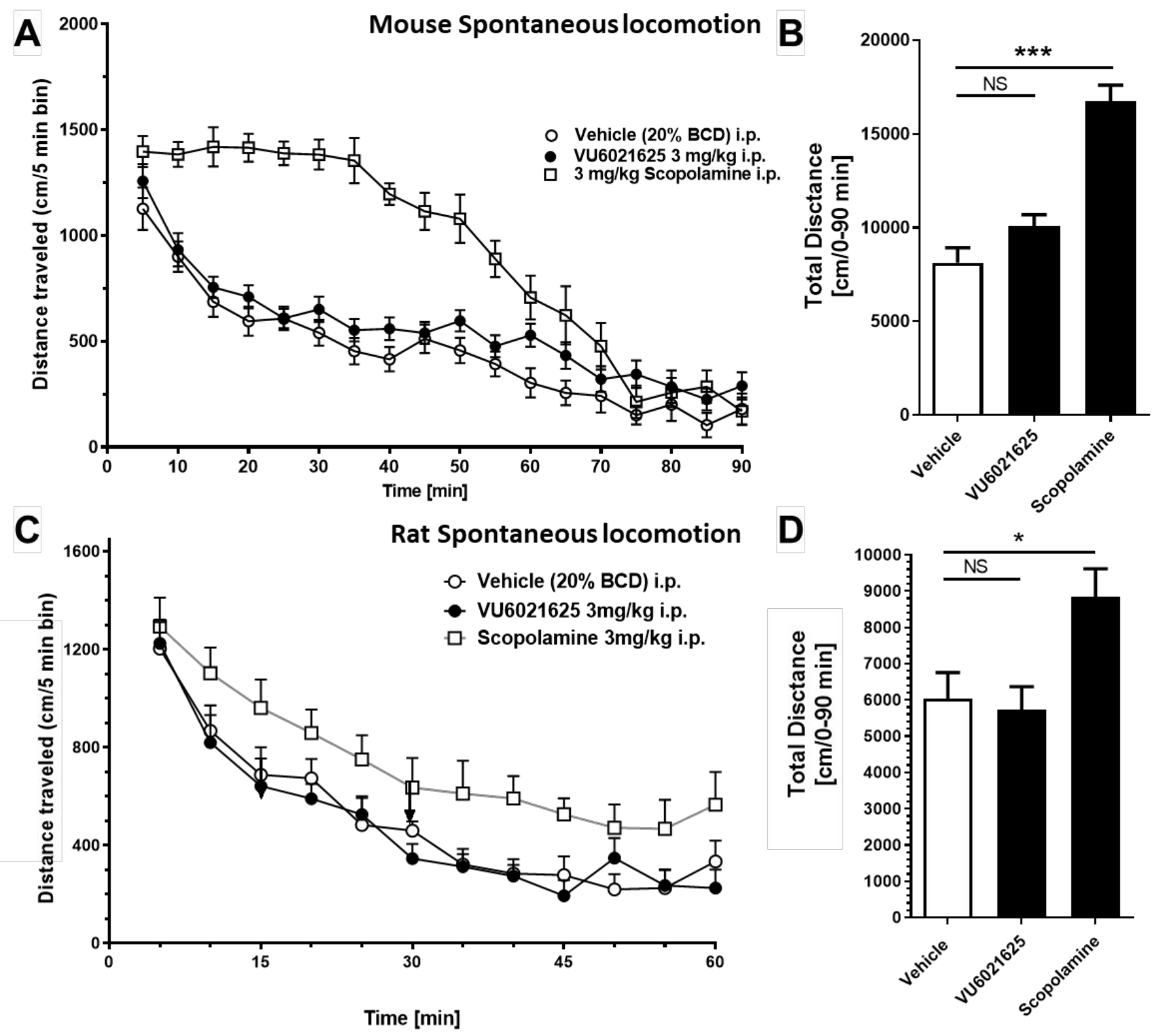

Figure S1. VU6021625 does not alter spontaneous locomotion. Unlike scopolamine (3 mg/kg, i.p.), VU6021625 (3 mg/kg, i.p.) does not increase spontaneous locomotor activity in either mice (A-B) or rats (C-D), (A, C time activity curve; B, D total distance travelled after vehicle, VU6021625, or scopolamine administration). Mouse $\mathrm{N}=12$ per group. Rat $\mathrm{N}=8$ per group. One way ANOVA with Dunnett's post-hoc test $* * p<0.01, * * * p<0.001$, NS, not significant. 


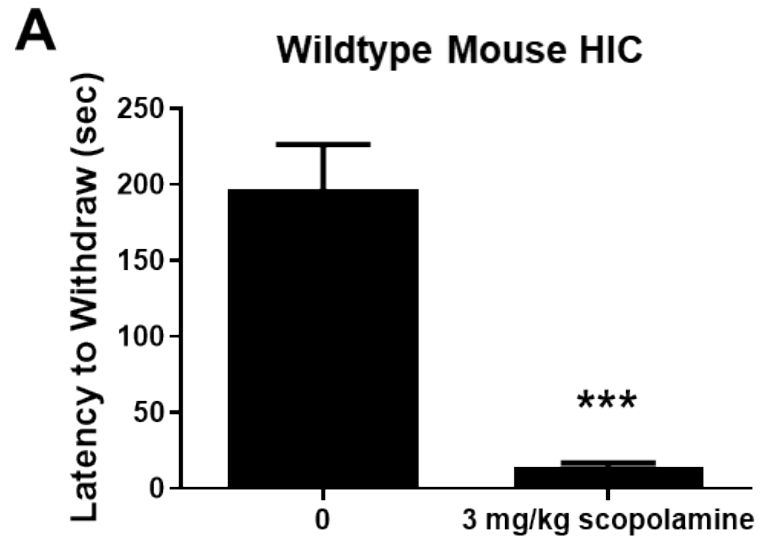

B

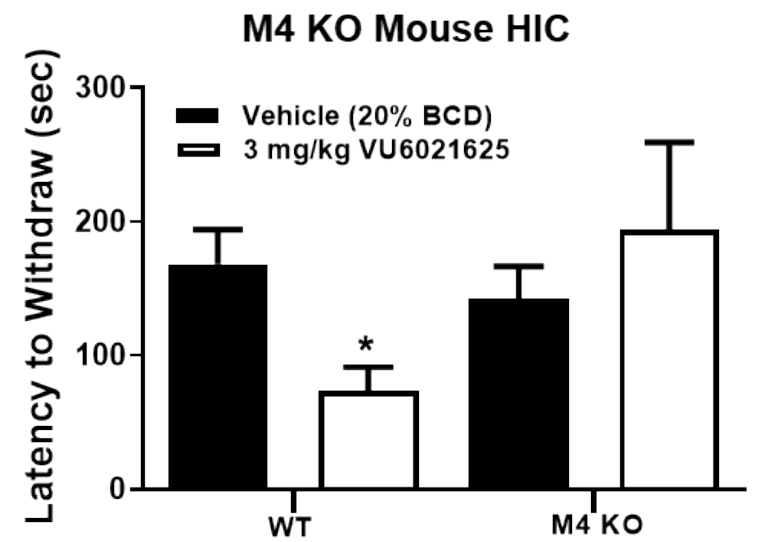

Figure S2. Wildtype and $\mathrm{M}_{4}$ knockout mouse haloperidol induced catalepsy. Comparison group of a maximally efficacious dose of scopolamine in reversing catalepsy (A). Systemic administration of VU6021625 demonstrates efficacy in reversing catalepsy in wildtype, but not $\mathrm{M}_{4}$ global knockout animals (B). $\mathrm{N}=8$ per group. Student's t test per. $\mathrm{N}=4-12$ per group. One way ANOVA with Dunnett's post-hoc test $* * \mathrm{p}<0.01,{ }^{* * *} \mathrm{p}<0.001, \mathrm{NS}$, not significant 